\title{
Structure of the quaternary complex of histone H3-H4 heterodimer with chaperone ASF1 and the replicative helicase subunit MCM2
}

\section{Dear Editor,}

The building block of eukaryotic chromatin is the nucleosome core particle (NCP), which is consisted of $\sim 146$ bps of DNA wrapped around an octamer of core histones. A tetramer of histone $\mathrm{H} 3$ and $\mathrm{H} 4$ and two $\mathrm{H} 2 \mathrm{~A}-\mathrm{H} 2 \mathrm{~B}$ dimers form the histone octamer (Kornberg, 1974; Luger et al., 1997; Thomas and Kornberg, 1975). During DNA replication, nucleosome disassembly and reassembly occurs at the replication fork, and histone chaperons CAF-1 and ASF1 are principally responsible for the deposition of histones $\mathrm{H} 3$ and $\mathrm{H} 4$ onto replicated DNA (Kaufman et al., 1995; Tyler et al., 1999). ASF1 binds a heterodimeric histone $\mathrm{H} 3-\mathrm{H} 4$ complex through its conserved $\mathrm{N}$-terminal domain (aa 1-155) and impedes the formation of the $(\mathrm{H} 3-\mathrm{H} 4)_{2}$ tetramer both inside the nucleus and in the cytoplasm (English et al., 2005). Humans have two ASF1 paralogs, ASF1a and ASF1b, both of which can be co-purified with the MCM 2-7 replicative helicase and histones $\mathrm{H} 3$ and $\mathrm{H} 4$ from HeLa cell nuclear extract, while only the MCM2 subunit can be pulled down by ASF1a or ASF $1 \mathrm{~b}$ together with $\mathrm{H} 3$ and $\mathrm{H} 4$ when using cytosolic extract of the same cells (Groth et al., 2007). In vitro studies showed that an N-terminal of human MCM2 (aa 63-154) binds histone $\mathrm{H} 3$ directly through a conserved motif (Foltman et al., 2013; Ishimi et al., 1998). The crystal structure of the MCM2 fragment in complex with the tetrameric $(\mathrm{H} 3-\mathrm{H} 4)_{2}$ complex has recently determined (Richet et al., 2015). However, it remains unknown whether $\mathrm{MCM} 2$ can bind histone $\mathrm{H} 3$ and $\mathrm{H} 4$ together with ASF1, and if so, whether the two bind in a synergistic manner or independently.

To answer these questions, we embarked on the characterization and structure determination of a quaternary complex of MCM2, ASF1 and histones $\mathrm{H} 3$ and H4. First, we find that they form an apparent 1:1:1:1 complex. The N-terminal domain of human MCM2 (aa 63-154), the globular domain of ASF1a (aa 1-157) and full-length human histones $\mathrm{H} 3$ and $\mathrm{H} 4$ were expressed in E. coli. A stable complex of the four proteins was obtained at a salt concentration of $0.5 \mathrm{~mol} / \mathrm{L} \mathrm{NaCl}$ (Fig. 1A). The tetrameric complex eluded from a Superdex 200 10/300 GL size exclusion column (GE Healthcare) at an elution volume of $14.38 \mathrm{~mL}$ (Fig. S1), corresponding to an apparent molecular weight of about $60 \mathrm{kDa}$, which is compatible with a stoichiometry of $1: 1: 1: 1$ of the four components. We then crystallized and solved a 3.5- $\AA$ structure of the quaternary complex, and the structure was solved by molecular replacement (see Supplemental Material for details). There are six MCM2-ASF1-H3-H4 tetramers in one asymmetric unit, the inter-tetramer contacts appear to be non-physiological, and we will concentrate our analyses on one tetramer henceforth.

The H3- $\mathrm{H} 4$ heterodimer is centrally located in the quaternary complex, enclosed on one side by MCM2 and the opposite side by ASF1 (Fig. 1B). MCM2 is composed of an $\mathrm{N}$-terminal tail followed by a short helix ( $\alpha 1)$, which is connected to a C-terminal helix ( $\alpha 2$ ) via a long loop. The extensive interaction between MCM2 and $\mathrm{H} 3-\mathrm{H} 4$ buries a total surface area of $1918 \AA^{2}$. The long loop snakes through the convex surface of the $\mathrm{H} 3-\mathrm{H} 4$ dimer, accounting for $\sim 2 / 3$ of the interaction surface area with $\mathrm{H} 3-\mathrm{H} 4$. A comparison with the recently published structure of the $(\mathrm{MCM} 2-\mathrm{H} 3-\mathrm{H} 4)_{2}$ complex (PDB code: 4UUZ) reveals that MCM2 interacts with $\mathrm{H} 3-\mathrm{H} 4$ similarly in the two complexes (Fig. 1C) (Richet et al., 2015). The observed interactions between ASF1 and $\mathrm{H} 3-\mathrm{H} 4$, mainly those between the globular domain of ASF1 and the $\mathrm{C}$-terminal $\alpha 3$ helix and the loop region between $\alpha 2$ and $\alpha 3$ of histone $\mathrm{H} 3$, also conform to those observed in the ternary ASF1-H3-H4 complex (PDB code: 2HUE) (English et al., 2006; Natsume et al., 2007). Interesting differences occur at the $\mathrm{C}$-terminal end of ASF1 where 11 residues (aa 154-164) are disordered in our structure (Fig. 1D). This segment of ASF1 would bump into a 2 of MCM2 if it took the conformation of that in the ternary ASF1- $\mathrm{H} 3-\mathrm{H} 4$ complex. The projection of the C-terminal segment of ASF1 is largely determined by the position of the immediately $\mathrm{N}$-terminal strand $\beta 9$, which is stabilized by antiparallel pairing with the C-terminal $\beta$-strand $(\beta C)$ of histone $\mathrm{H} 4$ (Fig. 1B). It is interesting to note that while $\beta C$ is positioned similarly in our quaternary complex and the ASF1- $33-\mathrm{H} 4$ ternary complex, it contrasts sharply with that found in the $(\mathrm{MCM} 2-\mathrm{H} 3-\mathrm{H} 4)_{2}$ complex (Fig. 1E). The conformation of $\beta C$ in the latter complex (PDB code: 4UUE, shows in gray) is similar to that of the nucleosomal $\mathrm{H} 4$, although not being stabilized by 

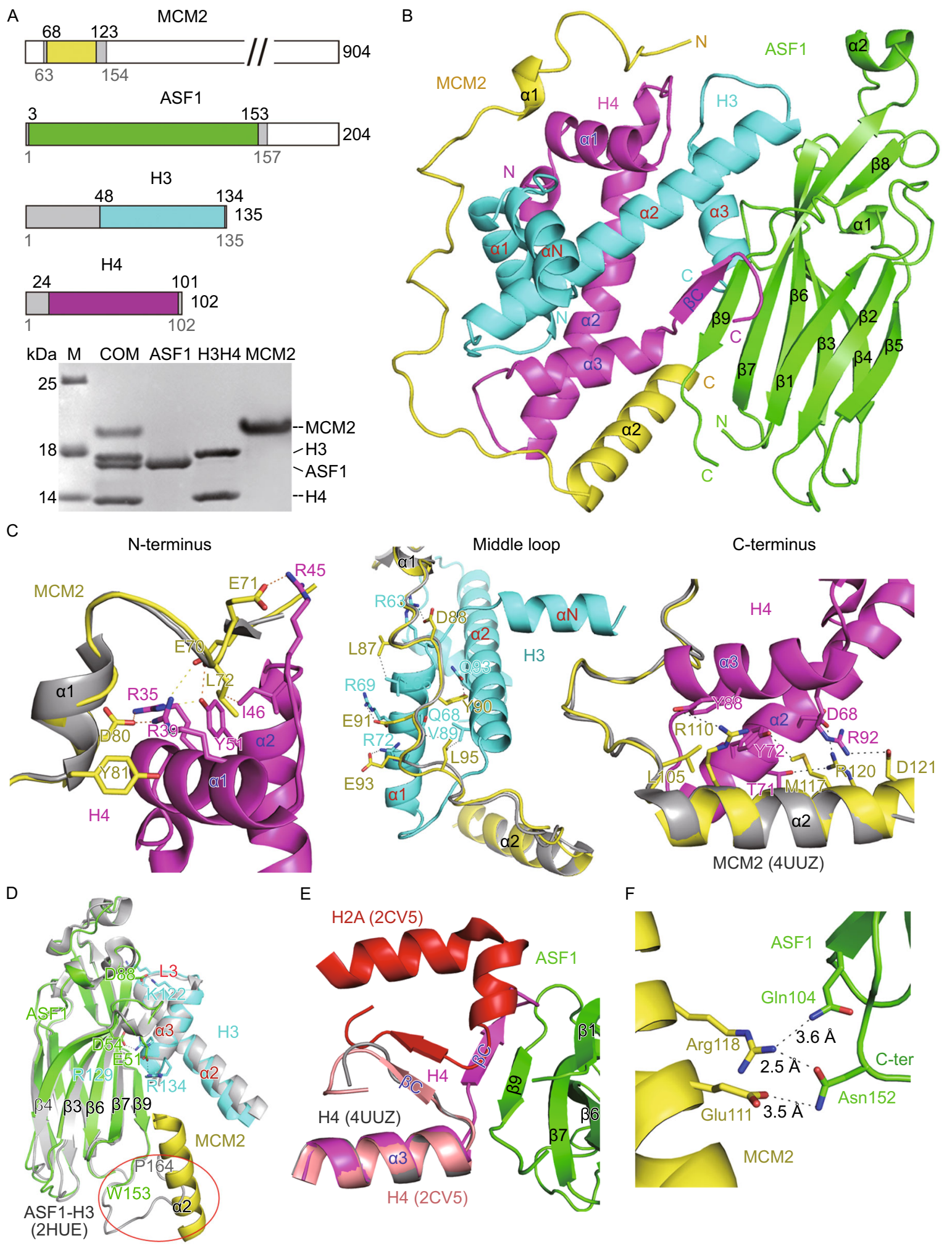
Figure 1. Structure of the MCM2-ASF1-H3-H4 complex. (A) Schematic diagram showing the truncated fragments of the four proteins. MCM2, ASF1, H3 and H4 are shown in yellow, green, cyan and magenta respectively (color coded the same in all figures). Disordered regions in the structure are shown in gray. An image of the coomassie-stained SDS-PAGE gel of the purified complex showing apparent stoichiometry of the four proteins. MCM2 appears to have an anomalous SDS-PAGE migration profile, as a Mass spectrometric measurement indicates a molecular weight 10,774 Da (data not shown). (B) A ribbon diagram showing the overall structure of the quaternary complex. (C) Interactions between MCM2 and H3$\mathrm{H} 4$ heterodimer. The structure of MCM2 from the ternary MCM2-H3-H4 structure (PDB code: 4UUZ, shown in gray) is superimposed for comparison. Three panels indicate $\mathrm{N}$-terminal, middle and C-terminal binding regions of MCM2. Residues involved in intermolecular interactions are shown in a stick model (carbon, yellow, magenta and cyan; nitrogen, blue; oxygen, red). Dashed lines indicate intermolecular hydrogen bonds. (D) Interactions between ASF1 and histone H3. Superposition of ASF1 and $\mathrm{H} 3$ from our quaternary structure and that from the ternary ASF1-H3-H4 structure (PDB code: 2HUE, shown in gray). C-terminal ends of ASF1 in the two structures and $\alpha 2$ MCM2 are highlighted inside the red circle. (E) Interactions between ASF1 and histone $\mathrm{H} 4$. The $\beta \mathrm{C}$ strand of $\mathrm{H} 4$ in the MCM2-H3-H4 ternary structure and in a human nucleosome structure (shown in gray and magenta, respectively) are aligned with that in the quaternary structure. Histone $\mathrm{H} 2 \mathrm{~A}$ in the nucleosome structure are shown in red. $(F)$ Direct interactions between MCM2 and ASF1.

pairing with the $\mathrm{C}$-terminal $\beta$ strand of histone $\mathrm{H} 2 \mathrm{~A}$ as in NCP (PDB code: 2CV5, H4 in pink and H2A in red) (Fig. 1E). Finally, although scarce, direct interactions between MCM2 and ASF1 are observed in the quaternary complex: the guanidine group of Arg118 of MCM2 forms a hydrogen bond with the hydroxyl group of Asn152 of ASF1; and weaker interactions between GIn104 and Asn152 of ASF1 and Arg118 and Glu111 of MCM2, respectively (Fig. 1F).

The principal functions of histone chaperones are to keep the histones in their pre-depositional, DNA-free state.
Superposition of our structure with the NCP structure (PDB code: $2 \mathrm{CV} 5$, shows in gray) via the $\mathrm{H} 3-\mathrm{H} 4$ heterodimer shows that (1) ASF1 occupies a position blocking the dimerization interface of histone $\mathrm{H} 3$, thus preventing the formation of a $(\mathrm{H} 3-\mathrm{H} 4)_{2}$ tetramer (Fig. 2A, Region 1); (2) MCM2 occludes the binding of histone $\mathrm{H} 2 \mathrm{~B}$ to histone $\mathrm{H} 4$ through its $\alpha 2$ helix, which impedes the association of $\mathrm{H} 2 \mathrm{~A}$ $\mathrm{H} 2 \mathrm{~B}$ heterodimers with $(\mathrm{H} 3-\mathrm{H} 4)_{2}$ tetramer to form an octamer (Fig. 2A, Region 2); (3) both the binding of MCM2 to the positively charged surface of the $\mathrm{H} 3-\mathrm{H} 4$ heterodimer and the protrusion of the C-terminal $\alpha 2$ helix of ASF1 would obstruct the wrapping of DNA in NCP. These features of MCM2 and ASF1 define them as bona fide histone $\mathrm{H} 3-\mathrm{H} 4$ chaperones.

Our structural study revealed the structural basis for the interaction between the ASF1-bound H3-H4 complex with MCM2. During replication-dependent or repair-coupled chromatin assembly, it is generally believed that ASF1 "hands" the $\mathrm{H} 3-\mathrm{H} 4$ heterodimer to the CAF-1 chaperonin complex prior to the deposition onto replicated DNA. Exactly how ASF1 is recruited to the replication or damage foci remains poorly understood, except that ASF1 is known to directly interact with the CAF-1 complex (Mello et al., 2002). $A$ recent result also showed the association of MCM complex with ASF1 in U2OS cells (Drissi et al., 2015). The interaction between MCM2 and the ASF1-H3-H4 complex may function together with $\mathrm{CAF}-1$ for efficient recruitment of the ASF1-H3-H4 complex, or alternatively, serves as an additional recruitment mechanism. As mentioned earlier, MCM2 co-purifies with ASF1 and histones $\mathrm{H} 3$ and $\mathrm{H} 4$ in the absence of other helicase components in the cytoplasm (Groth et al., 2007). Therefore, MCM2 appears to have a chaperoning function outside the context of a helicase complex, which functions exclusively in the nucleus. As with some other histone chaperones, such as NASP (Campos et al., 2010), the cytosolic MCM2 may also facilitate the nuclear import of histones $\mathrm{H} 3$ and $\mathrm{H} 4$. A summary of possible cellular functions of the interactions among $\mathrm{MCM} 2$, ASF1 and histones $\mathrm{H} 3$ and $\mathrm{H} 4$ is depicted in Fig. 2B, and the structural basis for their interactions learned here should help the dissection of their functions in chromatin biology.

The coordinates and structure factors of the MCM2ASF1-H3-H4 quaternary complex have been deposited in $\mathrm{PDB}$ under the accession code $5 \mathrm{C} 3 \mathrm{I}$. 

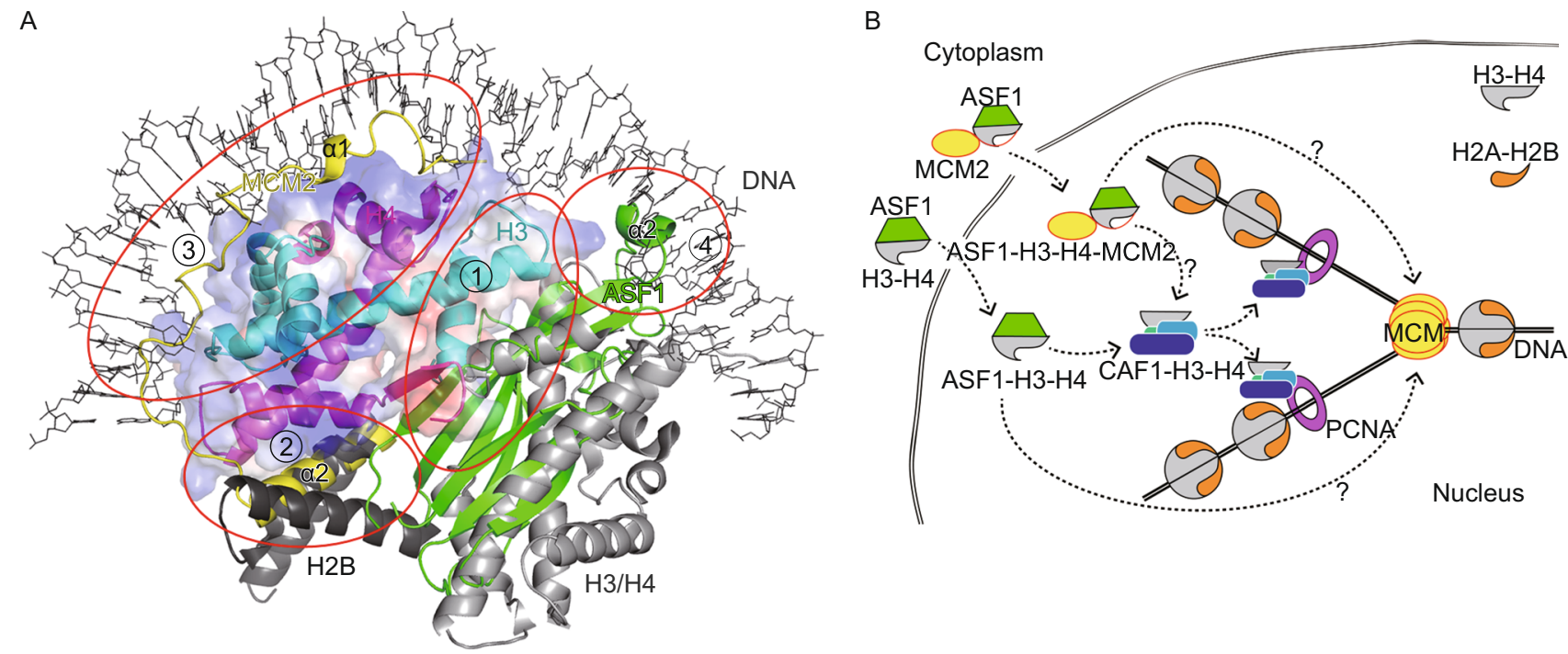

Figure 2. Possible biological functions of the MCM2-ASF1-H3-H4 complex. (A) The binding of MCM2 and ASF1 obstructs the formation of NCP and a histone $(\mathrm{H} 3-\mathrm{H} 4)_{2}$ tetramer. A human nucleosome structure (PDB code: $2 \mathrm{CV} 5$, shown in gray) is aligned with the MCM2-ASF1-H3-H4 structure via the H3-H4 heterodimer. Four obstructed regions are enclosed in red circles and numbered according to the order in which they were referenced in the text. (B) A model of possible biological functions of the MCM2-ASF1-H3$\mathrm{H} 4$ quaternary complex in the cytoplasm and in the nucleus. The cytosolic MCM2 may facilitate nuclear import of histones $\mathrm{H} 3$ and $\mathrm{H} 4$. In the nucleus, ASF1 "hands" the H3-H4 heterodimer to the CAF-1 complex prior to the deposition onto replicated DNA, in the presence or absence of MCM2. The ASF1-H3-H4 complex might also be directly recruited to the replication fork by interaction with the MCM complex.

\section{FOOTNOTES}

We thank SSRF beamline scientists for help with data collection and Qianglin Fang for participation at an early stage of the work. We also thank grant supports from the National Natural Science Foundation of China (Grant Nos. 31210103914 and 31430018 to R.M.X.), the National Basic Research Program (973 Program) (No. $2015 C B 856202$ to N.Y.), the Strategic Priority Research Program (XDB08010100) and the Key Research Program (KJZD-EW-L05) of Chinese Academy of Sciences (CAS). N.Y. is also supported by the Youth Innovation Promotion Association of CAS.

All authors declare that they have no conflict of interest. This article does not contain any studies with human or animal subjects performed by any of the authors.

Hong Wang ${ }^{1,2}$, Mingzhu Wang ${ }^{1}$, Na Yang ${ }^{1,2 \bowtie}$, Rui-Ming $\mathrm{Xu}^{1,2 \bowtie}$

${ }^{1}$ National Laboratory of Biomacromolecules, Institute of Biophysics, Chinese Academy of Sciences, Beijing 100101, China

2 University of Chinese Academy of Sciences, Beijing 100049 , China

$\triangle$ Correspondence: yangna@moon.ibp.ac.cn (N. Yang), rmxu@sun5.ibp.ac.cn (R.-M. Xu)

Electronic supplementary material The online version of this article (doi:10.1007/s13238-015-0190-0) contains supplementary material, which is available to authorized users.

\section{OPEN ACCESS}

This article is distributed under the terms of the Creative Commons Attribution 4.0 International License (http://creativecommons.org/ licenses/by/4.0/), which permits unrestricted use, distribution, and reproduction in any medium, provided you give appropriate credit to the original author(s) and the source, provide a link to the Creative Commons license, and indicate if changes were made.

\section{REFERENCES}

Campos El, Fillingham J, Li G, Zheng H, Voigt P, Kuo WH, Seepany H, Gao Z, Day LA, Greenblatt JF et al (2010) The program for processing newly synthesized histones H3.1 and H4. Nat Struct Mol Biol 17:1343-1351

Drissi R, Dubois ML, Douziech M, Boisvert FM (2015) Quantitative proteomics reveals dynamic interactions of the MCM complex in the cellular response to etoposide induced DNA damage. Mol Cell Proteomics, MCP

English CM, Maluf NK, Tripet B, Churchill ME, Tyler JK (2005) ASF1 binds to a heterodimer of histones $\mathrm{H} 3$ and $\mathrm{H} 4$ : a two-step mechanism for the assembly of the $\mathrm{H} 3-\mathrm{H} 4$ heterotetramer on DNA. Biochemistry 44:13673-13682

English CM, Adkins MW, Carson JJ, Churchill ME, Tyler JK (2006) Structural basis for the histone chaperone activity of Asf1. Cell 127:495-508

Foltman M, Evrin C, De Piccoli G, Jones RC, Edmondson RD, Katou Y, Nakato R, Shirahige K, Labib K (2013) Eukaryotic replisome 
components cooperate to process histones during chromosome replication. Cell Rep 3:892-904

Groth A, Corpet A, Cook AJ, Roche D, Bartek J, Lukas J, Almouzni $G$ (2007) Regulation of replication fork progression through histone supply and demand. Science 318:1928-1931

Ishimi Y, Komamura Y, You Z, Kimura H (1998) Biochemical function of mouse minichromosome maintenance 2 protein. J Biol Chem 273:8369-8375

Kaufman PD, Kobayashi R, Kessler N, Stillman B (1995) The p150 and $p 60$ subunits of chromatin assembly factor $\mathrm{I}$ : a molecular link between newly synthesized histones and DNA replication. Cell 81:1105-1114

Kornberg RD (1974) Chromatin structure: a repeating unit of histones and DNA. Science 184:868-871

Luger K, Mader AW, Richmond RK, Sargent DF, Richmond TJ (1997) Crystal structure of the nucleosome core particle at $2.8 \mathrm{~A}$ resolution. Nature 389:251-260
Mello JA, Sillje HHW, Roche DMJ, Kirschner DB, Nigg EA, Almouzni G (2002) Human Asf1 and CAF-1 interact and synergize in a repair-coupled nucleosome assembly pathway. EMBO Rep 3:329-334

Natsume R, Eitoku M, Akai Y, Sano N, Horikoshi M, Senda T (2007) Structure and function of the histone chaperone CIA/ASF1 complexed with histones $\mathrm{H} 3$ and $\mathrm{H} 4$. Nature 446:338-341

Richet N, Liu D, Legrand P, Velours C, Corpet A, Gaubert A, Bakail M, Moal-Raisin G, Guerois R, Compper C et al (2015) Structural insight into how the human helicase subunit MCM2 may act as a histone chaperone together with ASF1 at the replication fork. Nucleic Acids Res 43:1905-1917

Thomas JO, Kornberg RD (1975) Cleavable cross-links in the analysis of histone-histone associations. FEBS Lett 58:353-358

Tyler JK, Adams CR, Chen SR, Kobayashi R, Kamakaka RT, Kadonaga JT (1999) The RCAF complex mediates chromatin assembly during DNA replication and repair. Nature 402:555-560 